\title{
Video Article \\ Modified Blood Collection from Tail Veins of Non-anesthetized Mice with a Vacuum Blood Collection System and Eyeglass Magnifier
}

\author{
Xiaoyan Liu* ${ }^{1}$, Huanhuan $\mathrm{Li}^{*}{ }^{1}$, Jie Wu ${ }^{1}$, Yijun Yu ${ }^{1}$, Mingjing Zhang ${ }^{1}$, Wusong Zou ${ }^{1}$, Ye Gu ${ }^{1}$ \\ ${ }^{1}$ Heart Center, Wuhan Fourth Hospital, Puai Hospital Affiliated to Tongji Medical College, Huazhong University of Science and Technology \\ * These authors contributed equally
}

Correspondence to: Wusong Zou at zouwusong@163.com, Ye Gu at yegu2003cn@163.com

URL: https://www.jove.com/video/59136

DOI: doi:10.3791/59136

Keywords: Behavior, Issue 144, mice, tail vein, non-anesthetized, blood sample collection, vacuum blood collection system, eyeglass magnifier

Date Published: 2/2/2019

Citation: Liu, X., Li, H., Wu, J., Yu, Y., Zhang, M., Zou, W., Gu, Y. Modified Blood Collection from Tail Veins of Non-anesthetized Mice with a Vacuum Blood Collection System and Eyeglass Magnifier. J. Vis. Exp. (144), e59136, doi:10.3791/59136 (2019).

\section{Abstract}

Blood sample collection is the basis of experimental animal research. It is of importance to obtain adequate blood samples for various research purposes. The tail veins of mice are small, and it is sometimes difficult to obtain the required blood volume by conventional puncture methods. This study investigates the superiority of repeated blood sample collection from tail veins of mice through use of a vacuum blood collection system and eyeglass magnifier (experimental group) compared to conventional blood sampling methods (conventional group), performed by beginners and experts, respectively. With the help of an eyeglass magnifier, a butterfly needle tip is inserted into the tail vein of each mouse in the experimental group. When the vein is penetrated successfully, a blood sample is collected in the vacuum collection tube by insertion of the rubber end of a butterfly needle into the vacuum blood collection tube. The plunger is then used to collect blood without the help of the eyeglass magnifier in the conventional group. Success rates of blood sample collection by the beginners and experts were shown to be $70 \%$ vs. $100 \%$ $(p<0.01)$ in the experimental group and $35 \%$ vs. $85 \%(p<0.01)$ in the conventional group. For both beginners and experts, puncture times required for obtaining required blood sample were significantly lower in the experimental group compared to the conventional group (2.40 \pm 0.75 vs. $2.90 \pm 0.31, p<0.05 ; 1.15 \pm 0.37$ vs. $1.55 \pm 0.76, p<0.05)$. In conclusion, the presented blood sampling technique is feasible and easy to practice and enables frequent sampling of adequate blood volumes from non-anesthetized mice.

\section{Video Link}

The video component of this article can be found at https://www.jove.com/video/59136/

\section{Introduction}

Blood sampling from animals involved in experiments is a basic research technique. There are some available techniques for blood collection from mice, including tail snips and puncturing of the heart, retro-orbital plexus, jugular vein, caudal vein, and vena cava. Ideally, blood should be collected in a minimally invasive manner, with minimal impact on the animal's health. However, the most commonly used techniques often inflict stress upon animals and can impact research outcomes ${ }^{1}$. Blood collection from the retro-orbital plexus can be used to obtain enough blood volume from mice ${ }^{2}$. However, it can result in severe tissue damage and does not allow for obtaining blood repeatedly in short time intervals ${ }^{3}$.

The caudal vein is a superior location for blood collection, which inflicts minimal injury upon mice. However, the tail veins of mice are thin, and it is sometimes difficult to obtain enough blood through the conventional puncture technique. In some cases, repeated punctures are required to obtain the desired blood volume. Anesthesia is also commonly recommended to facilitate blood sampling from the tail veins of mice. Moreover, a scalpel, straight edge razor, or sharp scissors may be needed to remove the ends of the tails to obtain the required blood samples ${ }^{4}$. We have previously reported successful blood collection from the tail veins of non-anaesthetized rats by the vacuum blood sample collection system, which reduced the risk of blood contamination and avoided the need for repeated punctures ${ }^{5}$. This study reports a similar blood collection method in non-anesthetized mice.

\section{Husbandry}

1. Use 12 week old Kunming mice. NOTE: We used mice $(n=40,20$ males, $37-46 \mathrm{~g}$, mean $42.38 \pm 2.39 \mathrm{~g})$ from the Experimental Animals Center of Tongji Medical College.

2. House the mice under standard conditions with free access to food and drinking water. Keep two mice in a $530 \mathrm{~cm}^{2} \mathrm{cage}$ with wood shaving bedding.

3. Maintain a room temperature between $21-23^{\circ} \mathrm{C}$ 
4. Feed mice with a normal salt diet $(0.3 \% \mathrm{NaCl})$ throughout the study.

\section{Blood sample collection}

1. Prepare the following equipment: vacuum tube $(1 \mathrm{~mL})$, butterfly needle, eyeglass magnifier, and plastic restraining holder. Place them on a sterile surface (Figure 1).

2. Place a mouse in a plastic restraining holder and wash its tail with warm water $\left(20-30{ }^{\circ} \mathrm{C}\right)$. Wipe the tail with $70 \%$ ethanol-saturated cotton balls to expand the vein. Select the right or left tail vein for blood sampling. Grasp the lower portion of the tail gently and keep the tail straight during blood sample collection.

3. Collect the blood. If comparing methods, collect blood in two groups: the "experimental" group using the procedure we have developed, presented below, and "conventional" group using a conventional method, also presented below.

1. Experimental collection:

1. Wear an eyeglass magnifier to improve the viewing for puncture of the tail vein. Insert the $22 \mathrm{G}$ butterfly needle tip into one of the lateral tail veins at a position approximately half the distance distally from the tip of the tail at an angel approximately $10^{\circ}$, moving towards the base of the tail for multiple samples.

2. Insert the rubber end of the butterfly needle into the vacuum blood collection tube to collect blood (Figure 2). NOTE: If blood stops flowing out during blood collection, the needle angle should be quickly adjusted. To avoid blood coagulation in the needle, another puncture position should be selected if blood stops flowing out after $15 \mathrm{~s}$.

2. Conventional method:

1. Insert the needle connected to a syringe into one of the lateral veins approximately one-third of the distance distally from the tip of the tail.

2. When blood appears in the hub, pull back the plunger slowly to collect blood (Figure 3) ${ }^{7}$.

NOTE: To further elucidate the effects of varying experience with blood collection, a beginner and expert were chosen to collect blood samples using experimental and conventional methods simultaneously.

4. After blood collection, remove the needle and press the puncture point to stop bleeding. Then, release the mouse from the plastic restraining holder and return the mouse to its cage.

NOTE: It has been reported that up to $10 \%$ of total blood volume can be safely removed from a healthy animal at 2 week intervals ${ }^{8}$, so about $175 \mu \mathrm{L}$ of blood was collected each time in accordance with ethical principles.

5. Use tubes with EDTA to collect plasma and use tubes without anticoagulants to collect serum. Gently invert the tube several times and put them on ice vertically.

6. Centrifuge the blood sample collection tubes in a refrigerated centrifuge at $1,000 \mathrm{xg}$ for $10 \mathrm{~min}$ to separate plasma and serum. NOTE: Successful blood collection is defined as obtaining a volume of $175 \mu \mathrm{L}$ each time. No more than three punctures should be attempted, and a failed blood collection is defined as a total blood volume of less than $175 \mu \mathrm{L}$ after the third puncture. The sampling duration is defined as the time from the tail vein puncture to removal of the needle after blood collection.

7. Collect blood twice at intervals of 2 weeks ${ }^{8}$.

\section{Statistical analysis}

1. Use commercially available statistical software for analysis. Present data as mean value \pm standard deviation, using $p<0.05$ as the cutoff for statistical significance.

\section{Representative Results}

\section{Body mass, blood collection volumes, and sampling durations of the two groups}

Blood samples were collected from 20 mice (10 males) twice at 2 week intervals in each group. The mean body mass of mice was similar between the experimental and conventional groups for beginners and experts, respectively $(42.40 \mathrm{~g} \pm 1.42 \mathrm{~g}$ vs. $42.65 \mathrm{~g} \pm 1.14 \mathrm{~g}, \mathrm{p}>0.05$; $42.55 \mathrm{~g} \pm 2.91 \mathrm{~g}$ vs. $43.20 \mathrm{~g} \pm 2.69 \mathrm{~g}, \mathrm{p}>0.05)$. Collected blood volumes and sampling durations were similar between the two groups in experts $(184.25 \mu \mathrm{L} \pm 11.95 \mu \mathrm{L}$ vs. $171.75 \mu \mathrm{L} \pm 25.61 \mu \mathrm{L}, \mathrm{p}>0.05 ; 1.85 \mathrm{~min} \pm 0.68 \mathrm{~min}$ vs. $2.17 \mathrm{~min} \pm 0.80 \mathrm{~min}, \mathrm{p}>0.05)$. However, higher collected blood volumes and shorter sampling durations were seen in the experimental group compared to the conventional group in beginners (172.00 $\mu \mathrm{L}$ $\pm 15.17 \mu \mathrm{L}$ vs. $148.50 \mu \mathrm{L} \pm 30.22 \mu \mathrm{L}, p<0.01 ; 3.11 \mathrm{~min} \pm 0.44 \mathrm{~min}$ vs. $4.08 \mathrm{~min} \pm 0.61 \mathrm{~min}, \mathrm{p}<0.01)$. Compared to beginners, experts collected higher blood volumes and showed lower sampling times using experimental and conventional methods $(184.25 \mu \mathrm{L} \pm 11.95 \mu \mathrm{L}$ vs. $172.00 \mu \mathrm{L} \pm$ $15.17 \mu \mathrm{L}, \mathrm{p}<0.01 ; 171.75 \mu \mathrm{L} \pm 25.61 \mu \mathrm{L}$ vs. $148.50 \mu \mathrm{L} \pm 30.22 \mu \mathrm{L}, \mathrm{p}<0.05 ; 1.85 \min \pm 0.68 \min$ vs. $3.11 \min \pm 0.44 \mathrm{~min}, p<0.01 ; 2.17 \min \pm$ $0.80 \mathrm{~min}$ vs. $4.08 \mathrm{~min} \pm 0.61 \mathrm{~min}, \mathrm{p}<0.05)$ (Table 1 ).

\section{Success rates and puncture times of the two groups}

The comparison of success rates between beginners and experts was $70 \%(14 / 20)$ vs. $100 \%(20 / 20)(p<0.01)$ in the experimental group and $35 \%(7 / 20)$ vs. $85 \%(17 / 20)(p<0.01)$ in the conventional group. Higher success rates were also seen in the experimental group compared to the conventional group in beginners [70\% (14/20) vs. 35\% (7/20), $p<0.05]$. In both beginners and experts, the number of punctures was significantly lower in the experimental group compared to the conventional group $(2.40 \pm 0.75$ vs. $2.90 \pm 0.31, p<0.05 ; 1.15 \pm 0.37$ vs. $1.55 \pm$ $0.76, p<0.05)$. Compared to beginners, lower puncture times for experts were observed using experimental and conventional methods. $(1.15 \pm$ 0.37 vs. $2.40 \pm 0.75, p<0.01 ; 1.55 \pm 0.76$ vs. $2.90 \pm 0.31, p<0.01)$ (Table 1). 


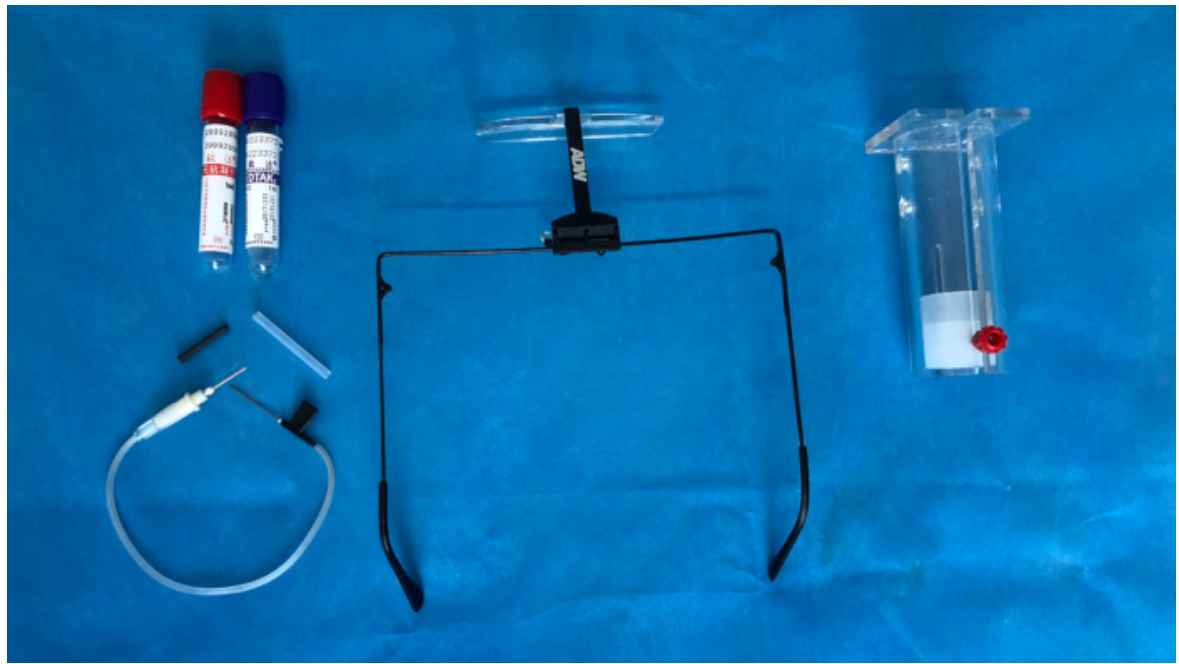

Figure 1: Equipment. Shown are $1 \mathrm{~mL}$ vacuum blood collection tubes and a $22 \mathrm{G}$ butterfly needle (left), an eyeglass magnifier (middle), and a plastic restraining holder (right). Please click here to view a larger version of this figure.

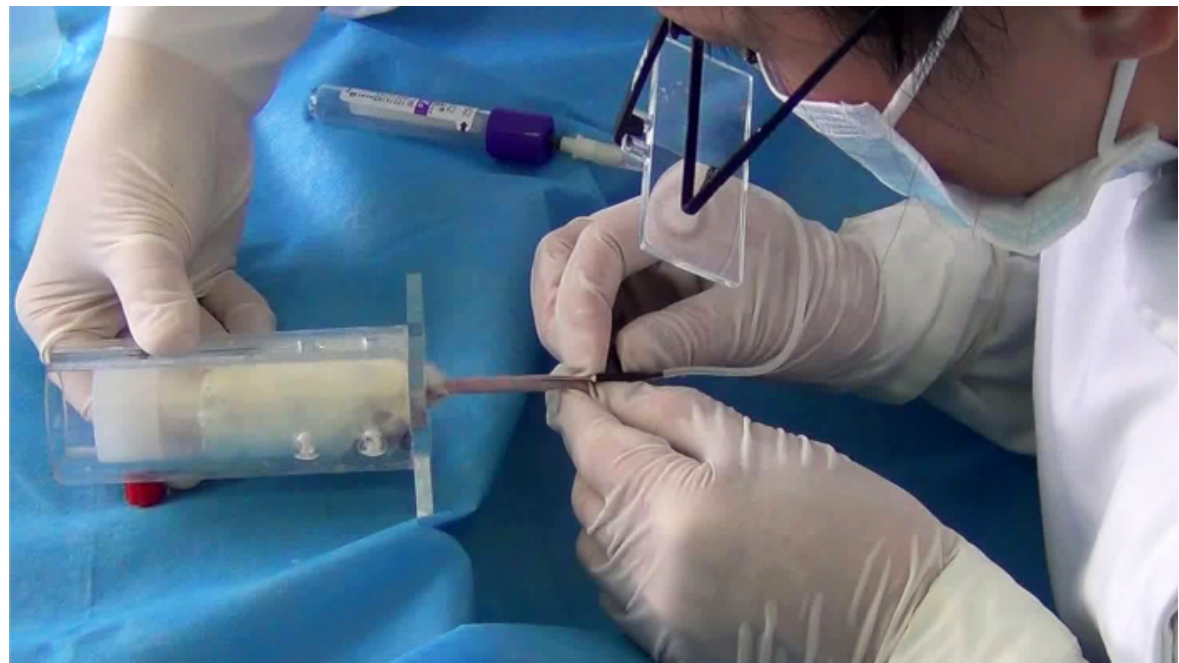

Figure 2: Successful blood collection in the experimental group. Please click here to view a larger version of this figure.

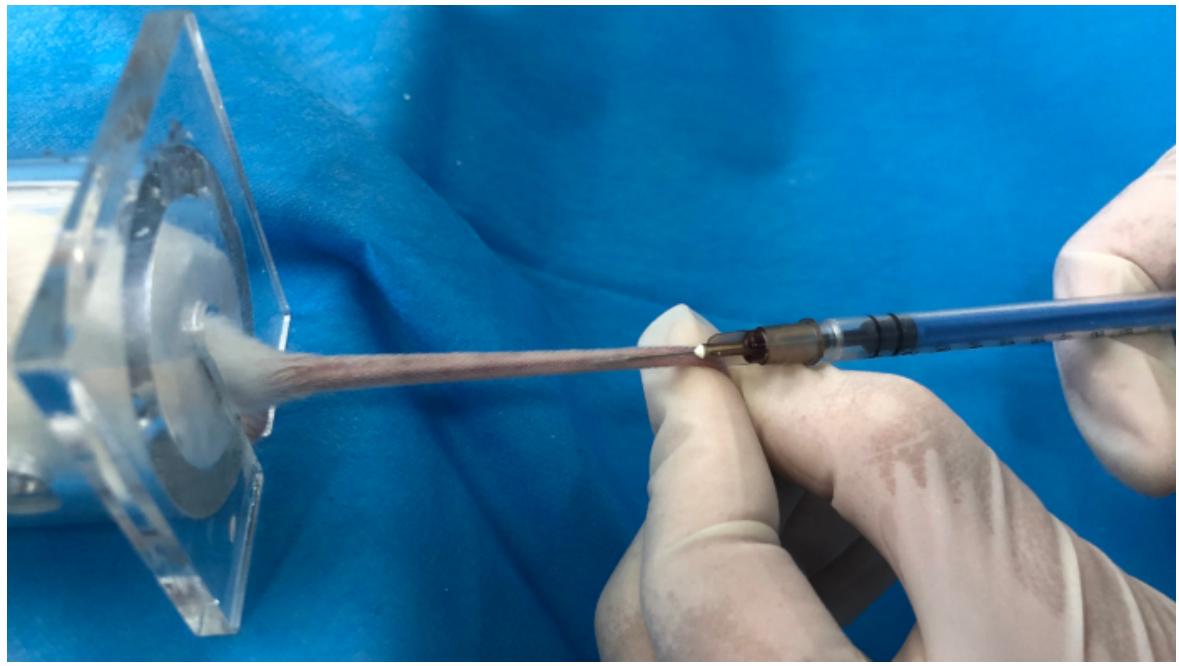

Figure 3: Successful blood collection in the conventional group. Please click here to view a larger version of this figure. 


\begin{tabular}{|l|l|l|l|l|}
\hline & \multicolumn{3}{|l|}{ Experimental group } & Conventional group \\
\hline & beginner & expert & beginner & expert \\
\hline Body mass $(\mathrm{g})$ & $42.4 \pm 1.42$ & $42.55 \pm 2.91$ & $42.65 \pm 1.14$ & $43.20 \pm 2.69$ \\
\hline $\begin{array}{l}\text { Collected blood volume } \\
(\mu \mathrm{L})\end{array}$ & $172.00 \pm 15.17$ & $184.25 \pm 11.95^{\# \#}$ & $171.75 \pm 25.61^{\#}$ \\
\hline Sampling duration (min) & $3.11 \pm 0.44$ & $1.85 \pm 0.68^{\# \#}$ & $4.50 \pm 30.22^{* *}$ & $2.17 \pm 0.80^{\# \#}$ \\
\hline Blood collection times & 20 & 20 & 20 & 20 \\
\hline $\begin{array}{l}\text { Average number of } \\
\text { punctures }\end{array}$ & $2.40 \pm 0.75$ & $1.15 \pm 0.37^{\# \#}$ & $1.55 \pm 0.76^{\# \#^{*}}$ \\
\hline One time puncture & 3 & 17 & $2.90 \pm 0.31^{*}$ & 12 \\
\hline Two times puncture & 6 & 3 & 0 & 5 \\
\hline Three times puncture & 5 & 0 & 2 & 5 \\
\hline Failed & 6 & 0 & 13 & 0 \\
\hline Success rate & $70 \%$ & $100 \%^{\# \#}$ & $35 \%^{*}$ & 3 \\
\hline
\end{tabular}

Table 1: Comparison of results between the experimental and conventional groups. ${ }^{*} p<0.05$, ${ }^{* *} p<0.01$, experimental method vs. conventional method. \#p $<0.05, \# p<0.01$, beginner vs. expert.

\section{Discussion}

The present study describes an easy-to-learn blood collection method in mice that is superior to the conventional techniques. First, the method can be easily mastered with a high success rate. Second, it is based on the vacuum negative pressure principle and allows for continuous drawing of blood with a reduced risk of direct blood exposure, which also reduces the chance of contamination and hemolysis ${ }^{9}$. Third, this method is feasible for frequent sampling of blood with adequate volumes from mice over a short period of time for various research purposes. Moreover, the procedure inflicts only minimal injury upon mice, and blood collection can be performed without the use of anesthetics; thus, the influence of the stress response and anesthetics on blood samples can be avoided.

The tail vein is a superior location for blood sampling according to the approved protocol ${ }^{7}$. However, it is not always easy to obtain sufficient blood volume from thin tail veins with low blood flows. In this case, the skin is usually cut open and vein is penetrated by a lancet, or the end of the tail is removed quickly by a razor.

This protocol aims to improve the methodology of blood collection from mice using the vacuum blood collection system, which requires a vacuum blood sample collection tube, butterfly needle, and eyeglass magnifier. This vacuum blood sampling system is usually used for collecting blood samples from patients in daily clinical practice ${ }^{10}$. With the help of an eyeglass magnifier, the perfect puncture point of a tail vein is easier to locate. When the tip of a needle is inserted into the tail vein, blood will automatically flow into the vacuum tube due to negative pressure. After withdrawing the needle from the tail vein, the blood that is blocked in the catheter will flow into the collecting vacuum tube due to the vacuum.

The following tips are important for successful application of the method. First, the body weight of each mouse should be $40 \mathrm{~g}$ or higher to decrease difficulty in puncturing and obtaining enough blood. Second, in the case of failed blood collection, experimenters should try to withdraw the needle slowly until blood continues to flow out. Third, it is vital to extend the tail to avoid any movement during blood sampling. Holding the needle gently can help to keep the needle tip in the vein as the mouse tail moves. Fourth, if blood stops flowing out during blood collection, the needle angle should be adjusted in a timely manner. In order to avoid blood coagulation in the needle, another puncture position should be selected if blood stops flowing out after $15 \mathrm{~s}$. Finally, the cooperation of two operators is recommended while using this technique to collect blood from mice.

In short, the adopted vacuum blood collection method for use in mice is safe, feasible, and easy to practice. This method enables frequent sampling of blood with adequate blood volumes from non-anesthetized mice.

\section{Disclosures}

The authors have nothing to disclose.

\section{Acknowledgments}

This work was supported by the Nature Science Foundation grant of Hubei Province, China [Grant No. 2018CFB761].

\section{References}

1. Grouzmann, E. et al. Blood sampling methodology is crucial for precise measurement of plasma catecholamines concentrations in mice. European Journal of Physiology. 447 (2), 254-258 (2003). 
2. Heimann, M., Roth, D. R., Ledieu, D., Pfister, R., Classen, W. Sublingual and submandibular blood collection in mice: a comparison of effects on body weight, food consumption and tissue damage. Lab Animal. 44 (4), 352-358 (2010).

3. Heimann, M., Kasermann, H. P., Pfister, R., Roth, D. R., Burki, K. Blood collection from the sublingual vein in mice and hamsters: a suitable alternative to retrobulbar technique that provides large volumes and minimizes tissue damage. Lab Animal. 43 (3), $255-260$ (2009).

4. Hoff, J. Methods of blood collection in the mouse. Lab Animal. 29 (10), 47-53 (2000).

5. Zou, W. et al. Repeated Blood Collection from Tail Vein of Non-Anesthetized Rats with a Vacuum Blood Collection System. Journal of Visualized Experiments. (130) (2017).

6. Kilkenny, C., Altman, D. G. Improving bioscience research reporting: ARRIVE-ing at a solution. Lab Animal. 44 (4), $377-378$ (2010).

7. Parasuraman, S., Raveendran, R., Kesavan, R. Blood sample collection in small laboratory animals. Journal of Pharmacology and Pharmacotherapeutics. 1 (2), 87-93 (2010).

8. Diehl, K. H. et al. A good practice guide to the administration of substances and removal of blood, including routes and volumes. Journal of Applied Toxicology. 21 (1), 15-23 (2001).

9. Wollowitz, A., Bijur, P. E., Esses, D., John Gallagher, E. Use of butterfly needles to draw blood is independently associated with marked reduction in hemolysis compared to intravenous catheter. Academic Emergency Medicine. 20 (11), 1151-1155 (2013).

10. Eder, J. M., Cutter, G. R. A new device for collecting cord blood. Obstetrics and Gynecology. 86 (5), 850-852 (1995). 